\title{
Estrategias didácticas y el rendimiento académico en los estudiantes de la Escuela de Ingeniería
}

\author{
Didactic strategies and academic performance in students of the School \\ of Industrial Engineering
}

\author{
- Carlos Ignacio Gallo Águila \\ cgalloa@gmail.com \\ Código ORCID: 0000-0003-1382-0545 \\ Universidad San Pedro, Perú
}

Artículo recibido en octubre 2020 / Arbitrado en noviembre 2020 / Publicado en enero 2021

Resumen La investigación aquí descrita tuvo como objetivo general interpretar la relación que existe entre las estrategias didácticas y el rendimiento académico en los estudiantes de la Escuela de Ingeniería Industrial en la Universidad San Pedro - filial Sullana. Considerando los aspectos planificación estratégica, conocimiento pedagógico y la comunicación didáctica. Se apoyó teórica y metodológicamente entre otros autores por: Rosales (2003), Ander (1995), Fernández (2006), Hernández (2010) y Bernal (2006) El enfoque metodológico fue cuantitativo - descriptivo y de tipo correlacional, empleando como técnica la entrevista y como instrumento el cuestionario. La población fue de 600 estudiantes y se trabajó con una muestra de 234. Se constató que existe una relación significativa entre las estrategias didácticas y el rendimiento académico en los estudiantes, porque los datos de la estadística descriptiva mostraron porcentajes altos y la estadística inferencial, adquirió un valor de significancia. Por lo tanto se rechazaron las hipótesis nulas y se aceptaron las hipótesis alternativas.

Palabras clave: Estrategias didácticas; rendimiento académico; universidad

\begin{abstract}
The research described here had the general objective of interpreting the relationship between didactic strategies and academic performance in students of the School of Industrial Engineering at San Pedro University - Sullana subsidiary. Considering the strategic planning, pedagogical knowledge and didactic communication aspects. Theoretically and methodologically supported among other authors by: Rosales (2003) Ander (1995) Fernández (2006) Hernández (2010) and Bernal (2006) The methodological approach was quantitative - descriptive and correlational, using the interview as a technique and as instrument the questionnaire. The population was 600 students and a sample of 234 was studied. It was found that there is a significant relationship between teaching strategies and academic performance in students, because the descriptive statistics data showed high percentages and the inferential statistics acquired a significance value. Therefore the null hypotheses were rejected and the alternative hypotheses were accepted.
\end{abstract}

Keywords: Didactic strategies; academic performance; university 
INTRODUCCIÓN

Esta investigación se situó en el escenario de una sociedad altamente tecnificada en la que los valores morales tradicionales, están abriendo paso a los valores emergentes referidos a la técnica y a la tecnología, como factores relevantes que intervienen el sistema educativo. Es en ese contexto donde las personas se relacionan de manera superficial, en el que el físico y el dinero constituyen la carta de presentación en una sociedad cada vez más convulsionada, con presencia de jóvenes que parecen rendir culto a la belleza externa, sin importar muchas veces el sentimiento del otro.

Este panorama se convierte en parte de las causas que desarrolla en los jóvenes un problema de rendimiento académico que los hace cada vez más débiles y los expone en una sociedad donde sobresale el más fuerte. Esto lleva a reflexionar a todos los comprometidos con la formación de los jóvenes, observando de cerca la frustración en sus rostros por no calzar en el estereotipo que les ha impuesto la sociedad y donde sus integrantes se ocupan de hacerlo saber y sentir en cada momento. Pero caben aquí las preguntas ¿qué hacen los padres? y ¿qué hacen los docentes? al respecto de este problema que en vez de formar deforma sentimientos y personalidades.

El presente trabajo tuvo como objetivo interpretar la relación entre las estrategias didácticas y el rendimiento académico en los estudiantes de la Escuela de Ingeniería Industrial en la Universidad San Pedro - filial Sullana.

El área problemática es lo referido al estudio de las estrategias didácticas que mejoran el rendimiento académico de los estudiantes de la Facultad de Ingeniería Industrial, por lo que se buscó determinar y explicar de manera particular en qué medida la aplicación de las estrategias didácticas que utiliza el docente influye en ese rendimiento académico.

Lo antes planteado no es ajeno en la institución educativa, pues se mostró este estudio y consideró que las estrategias didácticas empleadas por los docentes deben jugar un rol fundamental que no solamente permita rescatar el tiempo de los estudiantes y encaminarlo hacia objetivos definidos con anterioridad, sino que además posibilite generar las condiciones para el óptimo desarrollo de su rendimiento.

De esta manera, en la siguiente investigación se planteó la necesidad de investigar la relación que existe entre las estrategias empleadas por los docentes y el nivel de rendimiento académico de los estudiantes. En tal sentido se formuló la investigación en los términos siguientes.

La hipótesis estableció que existe una relación significativa entre las estrategias didácticas y el rendimiento académico en los estudiantes de la Escuela de Ingeniería Industrial en la Universidad San Pedro - filial Sullana.

El problema general se expresó en la siguiente interrogante:

¿Cuál es la relación entre las estrategias didácticas y el rendimiento académico en los estudiantes de la Escuela de Ingeniería Industrial en la Universidad San Pedro - filial Sullana?

\section{Problemas específicos}

1. ¿Cuál es la relación entre la planificación estratégica y el rendimiento académico en los estudiantes de la Escuela de Ingeniería Industrial? 
2. ¿Cuál es la relación entre el conocimiento pedagógico y el rendimiento académico en los estudiantes de la Escuela de Ingeniería Industrial?

3. ¿Cuál es la relación entre la comunicación didáctica y el rendimiento académico en los estudiantes de la Escuela de Ingeniería Industrial?

\section{Objetivo general}

Interpretar la relación que existe entre las estrategias didácticas y el rendimiento académico en los estudiantes de la Escuela de Ingeniería Industrial en la Universidad San Pedro - filial Sullana.

\section{Objetivos específicos}

1. Determinar la relación que existe entre la planificación estratégica y el rendimiento académico en los estudiantes de la Escuela de Ingeniería Industrial.

2. Describir la relación que existe entre el conocimiento pedagógico y el rendimiento académico en los estudiantes de la Escuela de Ingeniería Industrial.

3. Explicar la relación que existe entre la comunicación didáctica y el rendimiento académico en los estudiantes de la Escuela de Ingeniería Industrial.

La importancia de la presente investigación radicó en los resultados obtenidos y las aplicaciones posibles que de ella se desprendieron. Así se consideró que:

La importancia teórica radicó en el uso de la metodología científica de manera exhaustiva, lo cual permitió conocer mejor el estado de los conocimientos acerca de la temática abordada.
Se comprendió con mayor objetividad la influencia o relación que se ejerce entre las estrategias didácticas que emplearon los docentes y el rendimiento académico en los estudiantes de la muestra objeto de estudio.

La importancia práctica buscó tomar conciencia respecto a las estrategias didácticas empleadas por los docentes en el rendimiento académico de los estudiantes. Razón por la cual se realizó el estudio de las variables involucradas en esta acción pedagógica, puesto que de ellas dependerá la eficacia de la acción educativa y, por ende, el rendimiento de los estudiantes en general. Hecho que sin lugar a dudas redundará en el beneficio de la universidad y en el vínculo que ella ejerce con la labor diaria y la realización de las tareas en forma óptima. Brindando un servicio cada vez de mayor calidad, contribuyendo a su vez al mejoramiento de la calidad del servicio profesional del país.

La importancia social buscó responder a las necesidades sociales y culturales que la sociedad actual enfrenta, proporcionando elementos de juicio útiles, los cuales se constituyeron en referentes relevantes para optimizar el rendimiento académico, siendo un factor estratégico clave para el mejoramiento de la calidad educativa de los estudiantes.

\section{Estrategias didácticas}

En el ámbito educativo son los procedimientos que el alumno pone en marcha para concretar las capacidades propuestas en los objetivos de aprendizaje de sus programaciones de aula. Por lo tanto, las estrategias están integradas en el proceso de enseñanza - aprendizaje; de ahí que no deban trabajarse al margen del currículum, tal y como proponen, por ejemplo, los programas para 
enseñar a pensar. Las estrategias las emplea el profesor al enseñar y el alumno al aprender y si realmente son potentes y están bien ajustadas, las que se utilizan para transmitir información y para procesarla deben ser las mismas. Es decir, si se aprende mejor en red, se debe enseñar en red.

Las estrategias de enseñanza son todas aquellas ayudas planteadas por el docente que se proporcionan al estudiante para facilitar un procesamiento más profundo de la información. A saber, todos aquellos procedimientos o recursos utilizados por quien enseña para promover aprendizajes significativos. El énfasis se encuentra en el diseño, programación, elaboración y realización de los contenidos a aprender por vía oral o escrita.

Dichas estrategias deben ser diseñadas de tal manera que estimulen a los estudiantes a observar, analizar, opinar, formular hipótesis, buscar soluciones y descubrir el conocimiento por sí mismos. Organizar las clases como ambientes para que los estudiantes aprendan a aprender.

Rosales (2003) manifestó que el concepto de Estrategia (del latín strategema, y éste del griego strategía, de strategos, general jefe) proviene del ámbito militar "arte de proyectar y dirigir grandes operaciones militares, llevadas a cabo por los hoplitas (soldados griegos que llevaban armas pesadas)" (p.3). La actividad del estratega consistía en proyectar, ordenar y dirigir las operaciones militares para conseguir la victoria.

Ahora bien, en la intervención didáctica que realiza el docente en alguna asignatura, se ponen en juego infinitas variables. La complejidad de los procesos de enseñanza y aprendizaje, así como la inmensa diversidad de las situaciones en las que se desarrolla, hacen que la existencia de soluciones globales al problema de la enseñanza, "panaceas universales" de aplicación general, resulten absolutamente utópicas. Sin embargo, sí es posible enunciar las directrices, líneas maestras de actuación, sobre una serie de factores que intervienen en el proceso de enseñanza, así como proporcionar pautas para la utilización adecuada de una serie de estrategias didácticas.

Es importante a la hora de decidir el curso de acción, organizar situaciones de aprendizaje significativos que procuren cumplimentar con los propósitos previstos. Para ello, es necesario tener claro: ¿A dónde se quiere ir? ¿Cuál es el camino para alcanzar las expectativas fijadas? y ¿Cómo comprobar si se ha llegado a lo previsto? entre otras preguntas. Es por eso que el docente debe organizar, seleccionar y, por último, tomar decisiones que estarán mediatizadas por el modelo didáctico al cual se adhiera.

Toda práctica pedagógica tiene un supuesto básico subyacente, que se hace explícito en la forma de intervención docente durante las clases. El modo de actuar depende en gran medida de cómo se aprecia el mundo, la experiencia en el mundo físico, social y de las teorías personales.

En esta parte se da a conocer la importancia de las estrategias didácticas durante el proceso enseñanza - aprendizaje de la actividad educativa, con el fin de que las orientaciones del docente no queden solo en los contenidos y objetivos de los programas educativos. Para los estudiantes éstas estrategias juegan un papel protagónico en 
dicho proceso, debido a que ellos llegan a las instituciones universitarias con conocimientos previos acerca de hechos, circunstancias o realidades; ideas o pensamientos que pueden ser reforzados por el docente.

En este sentido las estrategias didácticas proporcionan las bases y elementos que permiten tanto al maestro como al alumno, obtener un determinado alcance en el desarrollo de competencias, ya que proporcionan información, desarrollan la motivación, establecen las técnicas de enseñanza y de aprendizaje que han de guiar el proceso educativo tanto dentro como fuera del aula. De igual manera, las estrategias orientan al profesor y al alumno, el camino que han de seguir para alcanzar la competencia en un nivel aceptable y por lógica alcanzar satisfactoriamente los objetivos establecidos en la planeación didáctica.

\section{Estrategias didácticas para promover aprendizajes significativos}

En el contexto educativo hoy casi no se habla ya de estímulo, respuesta, refuerzo positivo, objetivos operativos, instrucción programada y tecnología educativa. Estos conceptos forman parte del discurso usado en una época en la que la influencia comportamentalista en la educación estaba en auge, y se traducía explícitamente en las estrategias de enseñanza y en los materiales educativos. En esa época la enseñanza y el aprendizaje se enfocaban en términos de estímulos, respuestas y refuerzos, no de significados.

Actualmente las palabras en uso son aprendizaje significativo, cambio conceptual y constructivismo. Una buena enseñanza debe ser constructivista, promover el cambio conceptual y facilitar el aprendizaje significativo. Es probable que la práctica docente aún tenga mucho del conductivismo pero el discurso es cognitivista / constructivista / significativo. Lo que se quiere decir es que puede no haber aún un verdadero cambio conceptual en este sentido, pero parece que se está caminando en esa dirección (Moreira, 2000)

Si bien según el momento de su presentación en un episodio o secuencia de enseñanza-aprendizaje, las diferentes estrategias de enseñanza pueden incluirse al inicio (preinstruccionales) durante (coinstruccionales) o al término (posinstruccionales) Para la presente investigación se presentan como dimensiones para la construcción de las estrategias didácticas a la planificación estratégica, al conocimiento pedagógico y a la comunicación didáctica.

\section{Planificación estratégica}

La planificación se entiende como el diseño y la elaboración del currículo escolar en su globalidad. Etimológicamente programar se deriva del vocablo griego "prographo" que significa yo anuncio por escrito. Programar es la acción consistente en utilizar un conjunto de procedimientos mediante los que se organizan de manera racional y organizada una serie de actividades y acciones previstas de antemano, con las que se pretenden alcanzar determinadas metas y objetivos utilizando determinados recursos (Ander, 1995)

La planificación del curriculum ha de entenderse como un proceso a través del cual se toman las decisiones respecto al qué, para qué, cómo, cuándo dónde, en cuánto tiempo se pretende enseñar la materia. Es la toma de 
decisiones curriculares donde también está comprendida la forma cómo se evaluará, que corresponde a la pregunta ¿En qué medida se están logrando o se lograron los objetivos propuestos? Como se puede inferir, la planificación es una práctica en la que se delibera sobre diversas opciones, considerando las circunstancias específicas en las que se llevará a cabo (MINEDUC, Orientaciones, 2004)

Según Ander (1989):

Planificar es la acción consistente en utilizar un conjunto de procedimientos mediante los cuales se introduce una mayor racionalidad y organización en unas acciones y actividades previstas de antemano con las que se pretende alcanzar determinados objetivos, habida cuenta de la limitación de los medios (p.13)

La planificación es un proceso secuencial a través del cual se establecen una serie de pasos que conducen la enseñanza a una meta final. Una planificación eficaz requiere poner en marcha una serie de habilidades cognitivas, que no siempre resultan conscientes para el que planifica.

\section{Conocimiento pedagógico}

Si bien el docente debe ser un profesional preparado, conocedor del amplio espectro que constituye la materia que imparte, se debe destacar que en el momento actual no se puede seguir considerando a los docentes como almacenes del saber y por lo tanto dispensadores omnipotentes del conocimiento. La cantidad de información que existe sobre cualquier tema es de tal envergadura que es imposible pensar que puedan existir personas que pretendan saber todo de todo.

Al respecto Fernández (2006) indicó que en el momento que vivimos no basta con saber el contenido de la materia para enseñar bien. El profesor debe ser un conocedor de su materia, pero además ha de aprender a aprender, a ser un experto gestor de información sobre la misma, un buen administrador de los medios a su alcance y desde esta orientación dinamizar el aprendizaje de sus alumnos.

Desde este punto de vista el docente debe ser no solamente un profesional con preparación en la temática que le toca desarrollar, sino también con experiencia en este ámbito. Este aspecto le permitirá diseñar estrategias de enseñanza y aprendizaje para gestionar el desarrollo de las clases manteniendo el orden y motivando al alumnado (despertar la curiosidad y el interés, la participación y facilitar la comprensión de los contenidos básicos)

\section{Comunicación didáctica}

Uno de los aspectos más importantes de las relaciones entre las personas es la comunicación, ya que a través de ella logramos intercambiar ideas, experiencias y valores; transmitir sentimientos, actitudes y a conocernos mejor. La comunicación permite expresarse a las personas en el medio que les rodea.

Según Capote (2000) la comunicación didáctica en el aula se caracteriza por una relación terapéutica entre docente y estudiante constituyendo un auténtica encuentro entre 
seres humanos que luchan por la misma causa: la optimización de los aprendizajes. Si la relación es adecuada, el trabajo del docente es realizado más eficientemente y las situaciones son desarrolladas favorablemente. De esta manera una positiva relación entre docentes y estudiantes cultiva la efectividad en el proceso de aprendizaje

Dicha comunicación puede definirse como el proceso por medio del cual la información es intercambiada y entendida por un docente y uno o más estudiantes, usualmente con la intención en aquél de motivar o influir sobre las conductas de éstos, generándose así un encuentro donde no hay parte silenciosa.

\section{Rendimiento académico}

Omar y otros (2002) expresaron que en la vida académica, habilidad y esfuerzo no son sinónimos; el esfuerzo no garantiza un éxito y la habilidad empieza a cobrar mayor importancia. Esto se debe a cierta capacidad cognitiva que le permite al alumno hacer una elaboración mental de las implicaciones causales que tiene el manejo de las autopercepciones de habilidad y esfuerzo. Dichas autopercepciones si bien son complementarias, no presentan el mismo peso para el estudiante; de acuerdo con el modelo, percibirse como hábil (capaz) es el elemento central.

En este sentido, en el contexto escolar los profesores valoran más el esfuerzo que la habilidad. En otras palabras, mientras un estudiante espera ser reconocido por su capacidad (lo cual resulta importante para su estima) en el salón de clases se reconoce su esfuerzo. Si pretendemos conceptualizar el rendimiento académico a partir de su evaluación, es necesario considerar no solamente el desempeño individual del estudiante sino la manera como es influido por el grupo de pares, el aula o el propio contexto educativo.

En este sentido, Navarro (2000) en su estudio denominado Factores asociados al rendimiento escolar, refirió que se necesita conocer qué variables inciden o explican el nivel de distribución de los aprendizajes, los resultados de su investigación plantean que:

...las expectativas de familia, docentes y los mismos alumnos con relación a los logros en el aprendizaje reviste especial interés porque pone al descubierto el efecto de un conjunto de prejuicios, actitudes y conductas que pueden resultar beneficiosos 0 desventajosos en la tarea escolar y sus resultados...asimismo que: el rendimiento de los alumnos es mejor, cuando los maestros manifiestan que el nivel de desempeño y de comportamientos escolares del grupo es adecuado (s.n.)

Sin embargo, en su estudio Análisis de las calificaciones escolares como criterio de rendimiento académico, Cascón (2000) atribuyó la importancia del tema a dos razones principales:

Uno de los problemas sociales y no solo académicos que están ocupando a los responsables políticos, profesionales de la educación, padres y madres de alumnos y a la ciudadanía en general, es la consecución de un sistema educativo efectivo y eficaz que proporcione a los alumnos el marco idóneo donde desarrollar sus potencialidades. 
En este sentido y en la práctica la totalidad en los países desarrollados y en vías de desarrollo el indicador del nivel educativo adquirido, ha sido, sigue y probablemente seguirá siendo las calificaciones escolares. A su vez, estas son reflejo de las evaluaciones y/o exámenes donde el alumno ha de demostrar sus conocimientos sobre las distintas áreas o materias, que el sistema considera necesarias y suficientes para su desarrollo como miembro activo de la sociedad.

\section{MÉTODO}

En este estudio se empleó el enfoque cuantitativo - descriptivo. Hernández (2010) afirmó:

El enfoque cuantitativo (que representa, como dijimos, un conjunto de procesos) es secuencial y probatorio. Cada etapa precede a la siguiente y no podemos "brincar" o eludir pasos. El orden es riguroso, aunque desde luego, podemos redefinir alguna fase. Parte de una idea... se derivan objetivos y preguntas de investigación, se revisa la literatura y se construye un marco o una perspectiva teórica. (p. 4)

Dadas las características de esta tesis el tipo fue correlacional. Bernal (2006) afirmo que las investigaciones correlacionales pretenden "examinar relaciones entre variables o sus resultados, pero en ningún momento explica que una sea la causa de la otra. En otras palabras, la correlación examina asociaciones pero no relaciones causales" (p. 113).
La población estuvo conformada por todos los 600 estudiantes de la Escuela de Ingeniería Industrial de la Universidad San Pedro - filial Sullana y la muestra fue de 234. La técnica y el instrumento para la recolección de la información fueron la entrevista y el cuestionario. Compuesta por varias preguntas escritas para que el encuestado lea y responda igualmente por escrito. Bernal (2006) dijo que "es una de las técnicas de recolección de información más usadas, a pesar de que cada vez pierde mayor credibilidad por el sesgo de las personas encuestadas" (p. 177)

La investigación optó por la validez de contenido para la validación de los instrumentos. La validación de contenido se llevó a cabo por medio de la consulta a expertos. Al respecto, Hernández (2010) mencionó:

Otro tipo de validez que algunos autores consideran es la validez de expertos o face validity, la cual se refiere al grado en que aparentemente un instrumento de medición mide la variable en cuestión, de acuerdo con "voces calificadas". Se encuentra vinculada a la validez de contenido $y$, de hecho, se consideró por muchos años como parte de ésta. (p. 204)

\section{RESULTADOS}

A continuación se muestran las dimensiones de las estrategias didácticas caracterizadas en tres niveles para este estudio: la planificación estratégica, el conocimiento pedagógico y la comunicación didáctica. 
Tabla 1. Niveles de estrategia didácticas

\begin{tabular}{lrcccc}
\hline & Frecuencia & Porcentaje & $\begin{array}{c}\text { Porcentaje } \\
\text { válido }\end{array}$ & $\begin{array}{c}\text { Porcentaje } \\
\text { acumulado }\end{array}$ \\
\hline Válido & Bajo & 11 & 4,7 & 4,7 & 4,7 \\
& Medio & 172 & 73,5 & 73,5 & 78,2 \\
& Alto & 51 & 21,8 & 21,8 & 100,0 \\
& & & & & \\
& Total & $\mathbf{2 3 4}$ & $\mathbf{1 0 0 , 0}$ & $\mathbf{1 0 0 , 0}$ & \\
\hline
\end{tabular}

Figura 1. Nivel de planificación estratégica

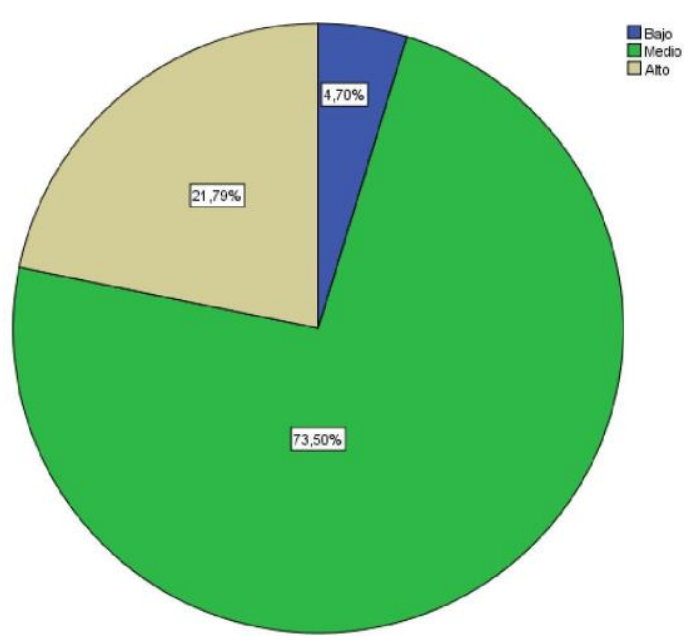

\section{Interpretación}

La tabla 14 y la figura 1 nos indican que el 21,79 \% de los datos se ubica en el nivel alto; seguido por el $73,5 \%$ que se ubica en el nivel medio; finalmente, un 4,7\% que se ubica en el nivel bajo. Estos datos son confirmados por los estadígrafos descriptivos correspondientes, en donde la mayoría tiene un nivel medio.

Tabla 2.Nivel de conocimiento pedagógico

\begin{tabular}{lccccc}
\hline & Frecuencia & Porcentaje & $\begin{array}{c}\text { Porcentaje } \\
\text { válido }\end{array}$ & $\begin{array}{c}\text { Porcentaje } \\
\text { acumulado }\end{array}$ \\
\hline Válido & Bajo & 10 & 4,3 & 4,3 & 4,3 \\
& Medio & 132 & 56,4 & 56,4 & 60,7 \\
& Alto & 92 & 39,3 & 39,3 & 100,0 \\
& Total & $\mathbf{2 3 4}$ & $\mathbf{1 0 0 , 0}$ & $\mathbf{1 0 0 , 0}$ & \\
\hline
\end{tabular}




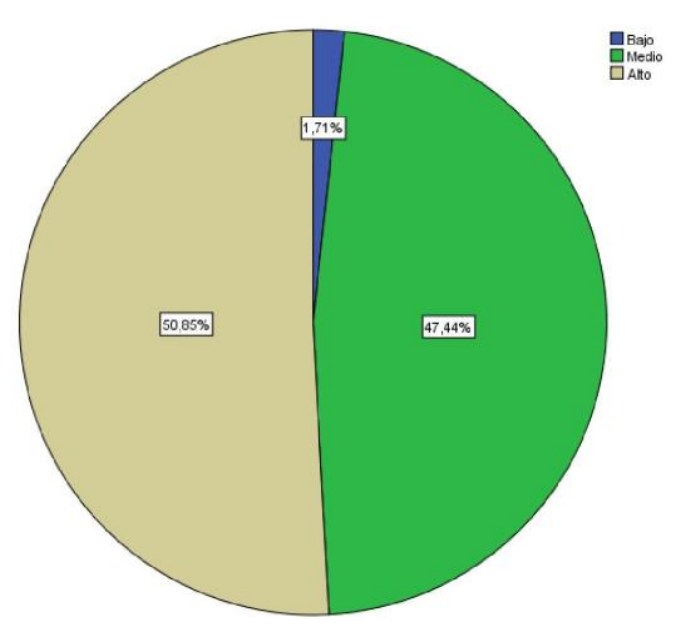

Figura 2. Nivel de conocimiento pedagógico

\section{Interpretación}

La tabla 15 y la figura 2 nos indican que el 39,3\% de los datos se ubica en el nivel alto; seguido por el $56,4 \%$ que se ubica en el nivel medio; finalmente, un $4,3 \%$ que se ubica en el nivel bajo. Estos datos son confirmados por los estadígrafos descriptivos correspondientes, en donde la mayoría tiene un nivel medio.

Tabla 3. Nivel de comunicación didáctica

\begin{tabular}{cccccc}
\hline & Frecuencia & Porcentaje & $\begin{array}{c}\text { Porcentaje } \\
\text { válido }\end{array}$ & $\begin{array}{c}\text { Porcentaje } \\
\text { acumulado }\end{array}$ \\
\hline Válido & Bajo & 4 & 1,7 & 1,7 & 1,7 \\
& Medio & 111 & 47,4 & 47,4 & 49,1 \\
& Alto & 119 & 50,9 & 50,9 & 100,0 \\
& Total & $\mathbf{2 3 4}$ & $\mathbf{1 0 0 , 0}$ & $\mathbf{1 0 0 , 0}$ & \\
\hline
\end{tabular}




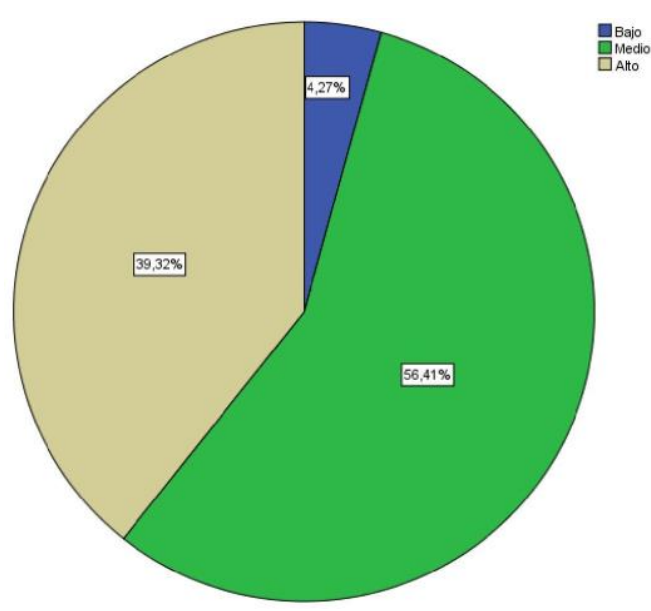

Figura 3. Nivel de comunicación didáctica

\section{Interpretación}

La tabla 16 y la figura 3 nos indican que el $50,9 \%$ de los datos se ubica en el nivel alto; seguido por el $47,4 \%$ que se ubica en el nivel medio; finalmente, un $1,7 \%$ que se ubica en el nivel bajo. Estos datos son confirmados por los estadígrafos descriptivos correspondientes, en donde la mayoría tiene un nivel alto.

\section{CONCLUSIONES}

Considerando el objetivo general interpretar la relación que existe entre las estrategias didácticas y el rendimiento académico en los estudiantes de la Escuela de Ingeniería Industrial en la Universidad San Pedro - filial Sullana. Se constató que existe una relación significativa entre las estrategias didácticas y el rendimiento académico en los estudiantes, porque los datos de la estadística descriptiva mostraron porcentajes altos y la estadística inferencial, adquirió un valor de significancia. Por lo tanto se rechazó la hipótesis nula y se aceptó la hipótesis alternativa.
De acuerdo con el primer objetivo específico: Determinar la relación que existe entre la planificación estratégica y el rendimiento académico en los estudiantes de la Escuela de Ingeniería Industrial. Se mostró que existe una relación entre la planificación estratégica y el rendimiento académico en los estudiantes porque los datos de la estadística descriptiva mostraron porcentajes altos y la estadística inferencial, adquirió un valor de significancia. Por lo tanto se rechazó la hipótesis nula y se aceptó la hipótesis alternativa.

Según el segundo objetivo: Describir la relación que existe entre el conocimiento pedagógico y el rendimiento académico en los estudiantes de la Escuela de Ingeniería Industrial. Se evidenció que existe una relación entre el conocimiento pedagógico y el rendimiento académico en los estudiantes porque los datos de la estadística descriptiva mostraron porcentajes altos y la estadística inferencial, adquirió un valor de significancia. Por lo tanto se rechazó la hipótesis nula y se aceptó la hipótesis alternativa. 
En concordancia con el tercer objetivo: Explicar la relación que existe entre la comunicación didáctica y el rendimiento académico en los estudiantes de la Escuela de Ingeniería Industrial. Se evidenció que existe una relación entre la comunicación didáctica y el rendimiento académico en los estudiantes porque los datos de la estadística descriptiva mostraron porcentajes altos y la estadística inferencial, adquirió un valor de significancia. Por lo tanto se rechazó la hipótesis nula y se aceptó la hipótesis alternativa.

Los docentes debieron enfatizar el desarrollo de estrategias didácticas para la adquisición de competencias para la elaboración de una adecuada planificación estratégica, promoviendo los conocimientos pedagógicos e informaciones de las distintas áreas y la comunicación didáctica. Si los estudiantes aprendiesen a adquirir información y a procesarla adecuadamente, entonces tendríamos un indicador más para el logro de la calidad educativa.

\section{REFERENCIAS}

Ander, E. (1989). Hacia una pedagogía autogestionaria. Buenos Aires: Lumen

Ander, E. (1995). Técnicas de investigación social. Buenos aires: Lumen

Ander, E. (1995). Introducción a la planificación. Buenos Aires: Lumen

Asociación de Psicólogos Americanos (2010) Manual de publicaciones de la American
Psychological Association. (3. ${ }^{\text {ra }}$ ed. en español). México: Manual Moderno

Bernal, C. (2006). Metodología de la investigación para administración, economía, humanidades y ciencias sociales ( $2^{\text {da }}$ ed.) México: Pearson

Cascón, I. (2000). Análisis de las calificaciones escolares como criterio de rendimiento académico. Recuperado de http://www3. usal.

es./inico/investigacion/jornadas/jornada2 /comunc/cl7. html

Fernández, H. (2006). Manual para elaborar investigaciones monográficas en educación. México: Limusa

Hernández, R.; Baptista, P. y Fernández, C. (2010) Metodología de la investigación. México: Mc Graw-Hill

MINEDUC. (2004). Orientaciones. Ministerio de Educación de Chile. Recuperado de www.mineduc.cl

Moreira, M. A. (2000). Aprendizaje significativo: teoría y práctica (pp. 3-100). Madrid: Visor

Navarro, R. E. (2003). Factores asociados al rendimiento académico. Revista iberoamericana de Educación, 33(1), 1-20

Omar, A.; Uribe, H.; Ferreira, M.C.; Leal E.M. y Terrones, A.J.M. (2002). Atribución Transcultural del Rendimiento Académico: Un Estudio entre Argentina, Brasil y México. Revista de la Sociedad Mexicana de Psicología, 17(2)

Rosales, C. (2003). Criterios para una Evaluación Formativa. Barcelona: Narcea 